Azctonurie) und im späteren Verlauf des Puerperiums mehr Fruchtbarkeitsstörungen (eitrige Genitalkatarrhe, Ovarialzysten, niedrige Erstbesamungsergebnisse) auf. Entsprechend waren auch die Abgänge wegen Sterilität und Stoffwechselstörungen in der Versuchgruppe 1 höher als in der Gruppe II. Der Verlauf der Erkrankungen war in der Gruppe I schlechter und erforderte mehr tierärztliche Behandlungen.

Die Versuchsergebnisse zeigen dass eine Uberversorgung an Energie und verdaul. Ronprotein in der Hochträchtigkeit zu einer starken Beeinträchtigung der Gesundheit und Fruchtbarkeit p.p. zur Folge haben kann. Der negative Einfluss kann sich bis etwa + Wochen p.p. auswirken. Die Versorgung sollte deshalb in der Hochträchtigkeit für die Erhaltung und 5 bis maximal ro $\mathrm{kg}$ Milch bemessen sein, um einen negativen Einfluss auf die Gesundheit und Fruchtbarkeit $z u$ vermeiden.

\title{
UNTERSUCHUNGEN ZUR ACETONÄMIE BEI DER MILCHKUH
}

\section{E. FARRIES, BRD.}

Acetonämie ist eine Störung im Energiestoffwechsel bei Hochleistungskühen zu Laktationsbeginn. Sie steht im Zusammenhang mit einer unvollständigen Metabolisierung von Fettreserven, die während der Gravidität angelegt wurden. Besondere Belastungen im Glukose-Stoffwechsel werden dabei beobachtet.

Versuchsanstellung : Durch gezielte Fütterungsmassnahmen wurden bei 60 Hochleistungskühen in der Spätgravidität (letzte Io Wochen a.p.) unterschiedliche Energiereserven angelegt, die dann zu Laktationsbeginn (erste ro Wochen p.p.) einer differenzierten Nutzung unterlagen.

Versuchsergebnisse : Gewichtsentwicklung : Die Gewichtsentwicklung als Mass für den V'erlaut von Reservebildung und Mobilisierung steht in deutlicher Abhängigkeit von der Nährstoffzufuhr. Ein Einfluss auf die Kälbergewichte ist kaum kaum zu erkennen.

Glukose : In der Gravidität bestehen keine fütterungsabhängigen I'nterschiede in der Blut-Glukose-Konzentration. Zu Laktationsbeginn liegt der Glukose-Spiegel bei den vorher hochversorgten Tieren deutlich niedriger ( $\mathrm{Ca} 40 \mathrm{mg} / 100 \mathrm{ml}$ ) als bei den niedrigversorgen ( $\mathrm{Ca} 50 \mathrm{mg} / \mathrm{IOO} \mathrm{ml}$ ). Ketonkörper: Die Konzentration an Ketonkörpern (Aceton, Acetacetat, $\beta$-Hydroxybutyrat) ist der den Glukose entgegengesetzt. Tiere mit hoher Energiereserve und verstärkter Mobilisierung erreichen hohe Werte (30 $\mathrm{mg} / \mathrm{r}$ oo $\mathrm{ml}$ ) gegenüber denen mit geringem Depot ( $\mathrm{I}+\mathrm{mg} / \mathrm{I}$ oo $\mathrm{ml}$ ).

Erhöhte Reservebikdung zum Zeitpunkt der Spätgravidität führt zu eine verstärkten Belastung des Energiestoffwechsels am Laktationsbeginn.

\section{VERWENDUNG VON STOFFWECHSELPARAMETERN ZUR BEURTEILUNG VON HALTUNGSSYSTEMEN BEIM RIND}

B. Oldigs, D. Smidt, H. J. Langholz, U. Meyer-Otting und G. Flitz-Pries. - - B B

Es wird über Untersuchungen an drei Kuhpopulationen in mehreren Haltungssystemen (Anbindestall, Kombibuctenstall, Tieflaufstall, Weide) berichtet. An Hand der ersten Versuchsergebnisse, die u.a. Stoffwechselparameter zum Inhalt hatten, wird diskutiert, ob bzw. welche Parameter zur Bewertung der Haltungssysteme geeignet sein können.

\section{THE INTERRELATIONSHIP BETWEEN METABOLIC PARAMETERS IN BLOOD SERUM OF CATTIE AND FERTILITY}

\author{
H. SOMmer. - Universität Bonn, Institute of Anatomy and Physiology and Hygiene, BDR.
}

Zwischen dem Gesamtcholesterin und der GOT im Blutserum bestehen Beziehungen zu verschiedenen Fruchtbarkeitsstörungen und damit zur Fruchtbarkeitsleistung. Die Verwendung der oben genannten und ähnlicher Parameter als Hilfsmerkmale zur Selektion auf Fruchtbarkeit 
stösst allerdings auf Schwierigkeiten, da die Merkmale im Blutserum wenig konstant sind. Im wesentlichen sind die Schwankungen durch das Grundfutter bedingt, wodurch z.T. Jahres. saisonale und betriebliche Unterschiede hervorgerufen werden. Die daraus entstehende Problematik wird am Beispiel mehrjähriger Untersuchungen erläutert.

\title{
ERBLICHKEITSSCHÄTZUNG FÜR VERSCHIEDENE METABOLITEN IM BLUTSERUM VON BULIEN
}

\section{N. H. FiSCHER. - Institut f. Tierhygiene Bonn, BRD.}

An I092 Bullen wurden folgende Serumbestandteile gemessen : die Aktivität der GlutamatOxalacetat Transaminase (GOT), das Gesamtcholesterin, die wahre Glucose, anorganisches Phosphat, Calcium, Natrium und Kalium. Bei allen Mineralstoffen zeigten sich hochsignifikante jahreszeitliche Unterschiede. Bei der GOT-Aktivität, dem Gesamtcholesterin und der GIucose war dieser Effekt nicht so ausgeprägt und regional unterschiedlich. Nur bei der GOT-Aktivität und der Glucose zeigten sich auch Unterschiede zwischen den Zuchtverbänden. Die Kovariablen Alter, Gewicht und durchschnittliche Milch-und Fettleistung der Mutter waren in ihrem Einfluss auf die Metaboliten sehr unterschiedlich.

Die geschätzten Heritabilitätskoeffizienten sind für : die GOT-Aktivität o, I 8 ; das Gesamtcholesterin 0,30 ; die Glucose 0,26 ; anorg. Phosphat o ; Calcium 0,33 ; Natrium o, I 2 ; und Kalium o. Die phänotypischen Korrelationen zwischen den Serumwerten waren unbedeutend.

Bej GOT zu Cholesterin, GOT zu Glucose und Cholesterin zu Calcium wurden genetische Korrelationen in der Grössenordnung von 0,4 geschätzt. Dabei war jedoch der Standardfehler sehr hoch, so dass keine eindeutige Beziehung angenommen werden kann.

\section{Systèmes planifiés de croisement chez le Porc}

\section{LES BASES DU CROISEMENT CHEZ I.E PORC}

\author{
P. Sellier. - Station de Génétique quantitative et appliquée, I. N. R. A., 78350 Jouy en Josas, \\ France.
}

Les différents paramètres du croisement sont passés en revue, dans,la ligne de l'analyse de Dickerson (1969). Le gain génétique résultant du croisement a une double origine : complémentarité, hétérosis. L'importance pratique de chacun de ces effets est évaluée, dans le cas du Porc. Des valeurs moyennes de l'hétérosis individuel et de l'hétérosis maternel pour les principaux caractères sont déduites d'estimations expérimentales présentées sous forme graphique. Il apparaît que, chez le Porc, les effets d'hétérosis, dont la part exploitée dans différents plans de croisement est rappelée, expliquent la majeure partie du gain lié au croisement. La variation observée des estimées d'hétérosis pour un caractère est analysée : variance d'échantillonnage, aptitude spécifique à la combinaison de certains couples de races, interactions de type hétérosis $\times$ milieu liaison entre hétérosis et moyenne parentale; quelques illustrations sont données. Enfin les différents critères utilisés en matière d'optimisation des stratégies de croisement chez le Porc sont présentés : la tendance actuelle est de ne plus rechercher seulement la productivité maximum de l'étage terminal du plan de croisement mais de s'intéresser au système de croisement dans son ensemble, soit à l'équilibre démographique, soit dans la phase de mise en place. Les premiers résultats d'une telle étude prévisionnelle concernant deux races françaises sont donnés.

\section{The EVAluation OF HYBRID PIGS}

D. M. Smith, D. E. Steane. - Meat and Livestock commission, Bletchley, Buckinghamshive, England.

British hybrid pig testing, known as Commercial Product Evaluation, aims to provide an independent assessment of stock offered for sale by breeding companies. In 1972-73 pigs were purchased from eight commercial companies, together with pure Large White and unimproved 